\title{
On Interpolation and Resampling of Discrete Data
}

\author{
Pouya Dehghani Tafti, Student Member, IEEE, Shahram Shirani, Senior Member, IEEE, and \\ Xiaolin Wu, Senior Member, IEEE
}

\begin{abstract}
This letter introduces a new representation of discrete signals based on the mathematical notions of functionals and continuous dual spaces. A new and more general sampling theorem is also suggested. Next, the problems of interpolating and resampling discrete signals are addressed; and a general solution using functional interpolation-which is applicable to many different settings-is proposed. Families of resampling filters dubbed de Boor-Ron filters that use de Boor-Ron interpolation are introduced, and their numerical realization is discussed. Some applications of this research are suggested.
\end{abstract}

Index Terms-de Boor-Ron filters, image interpolation, multiresolution, multivariate interpolation, resampling, sampling theorem, super-resolution, wavelets.

\section{INTRODUCTION}

$\mathbf{T}$ HE need to resample regularly or irregularly distributed discrete data arises in many different situations. For instance, measurements of a meteorological observable (temperature, pressure, humidity, etc.) are usually made at irregularly distributed locations. For ease of computation and analysis, one may wish to resample the data onto a regular grid. Other examples are the problems of demosaicing (de-mosaic-ing) and resolution enhancement in video processing (see [1], [2], and references therein), where to improve the color fidelity and spatial resolution of images, multiple video frames can be superimposed using real-valued motion vectors to form an irregular pixel grid of higher density. A high-resolution image can then be formed by resampling from this irregular pixel grid. These are only some of the many applications where one wants to resample discrete data provided as irregularly distributed measurements (samples). For such applications, it is important to design interpolation and resampling schemes with attention to the nature of the sampling process.

Resampling also provides a means of changing the density of samples. It can produce approximations at either lower or higher resolutions than that of the original data, thus leading to multiresolution signal processing schemes (for two examples of schemes based on resampling, see [3] and [4]).

In this letter, we introduce a new class of filters (which we name after C. de Boor and A. Ron) that is suitable for resampling discrete data obtained by (usually irregular) sampling of a function defined over a domain in $\mathbb{R}^{d}, d \geq 1$. By basing our

Manuscript received February 8, 2006; revised April 16, 2006. The associate editor coordinating the review of this manuscript and approving it for publication was Dr. Zhou Wang.

P. D. Tafti was with the Department of Electrical and Computer Engineering, McMaster University, Hamilton, ON L8S 4K1, Canada and is now with the Biomedical Imaging Group, EPFL/STI/IOA, CH-1015 Lausanne, Switzerland (e-mail: p.d.tafti@ieee.org).

S. Shirani and X. Wu are with the Department of Electrical and Computer Engineering, McMaster University, Hamilton, ON L8S 4K1, Canada (e-mail: shirani@ece.mcmaster.ca; xwu@ece.mcmaster.ca).

Digital Object Identifier 10.1109/LSP.2006.879854 design on functional interpolation, we deal not only with irregularities in the locations of the sampling points but also with differences in the nature and underlying physics of samples, in a natural and effective manner.

The framework we introduce is based on a novel understanding of sampling, which uses the notions of functionals and continuous dual spaces. Due to its suitability and usefulness in a wide range of problems, we employ functional interpolation using multivariate polynomials and in particular use de Boor and Ron's solution to the interpolation problem (cf. [5]-[8]). However, the same approach can also be applied using other classes of functions as interpolants.

In the following, after briefly reviewing some preliminary mathematical definitions, we formally define sampling and discrete signals in Section II. We then continue to introduce de Boor-Ron resampling filters in Section III and next provide some generic examples in Section IV. Implementation details and a few remarks then conclude this letter.

\section{SAMPLING AND DisCRETE SIGNALS}

\section{A. Notation and Mathematical Preliminaries}

We briefly recall several notions from functional analysis. The reader may refer to [9] and [10] for detailed treatments.

Let $\mathbb{F}$ denote a field (e.g., $\mathbb{R}$ or $\mathbb{C}$ ). Also let $\mathcal{X}$ be a topological vector space over the field $\mathbb{F}$, and let $f, g \in \mathcal{X}$. Then, we have the following.

Definition 1: A functional $\lambda$ on $\mathcal{X}$ is a map $\lambda: \mathcal{X} \longrightarrow \mathbb{F}$. It is linear if $\lambda(c f+g)=c \lambda(f)+\lambda(g)$ for all $f, g \in \mathcal{X}, c \in \mathbb{F}$. $\lambda f$ is commonly used in place of $\lambda(f)$ to denote the value assigned to $f$ by $\lambda$. The vector space of all continuous linear functionals on $\mathcal{X}$ is called the continuous dual of $\mathcal{X}$ and is denoted here by $\mathcal{X}^{*}$.

Example: When $\mathcal{X}$ consists of continuous scalar-valued functions defined over a domain $\Omega$, the point-evaluation functional at $\theta \in \Omega$ is the continuous linear functional $\delta_{\theta}: f \mapsto f(\theta)$.

Example: For a finite measure $m$ on $\Omega$ and a space $\mathcal{X}$ of $m$-measurable functions, the integral $\mu f:=\int_{\Omega} f \mathrm{~d} m$ defines a continuous linear functional $\mu$, which corresponds to averaging with respect to the measure $m$.

Definition 2: The closure of a set $A \subset \mathcal{X}$, denoted by $\bar{A}$, is the smallest closed set that contains $A$. $A$ is said to be dense in $B \supset A$ if $B \subset \bar{A}$.

Example: The closure of the set of rationals, $\mathbb{Q}$, in $\mathbb{R}$ (with its usual topology) is equal to $\mathbb{R}$ itself. Hence, $\mathbb{Q}$ is dense in $\mathbb{R}$.

Definition 3: A set $\Lambda \subset \mathcal{X}^{*}$ is weakly-* dense in $\mathcal{X}^{*}$ if it is dense in $\mathcal{X}^{*}$ with respect to the weak-* (pronounced weakstar) topology, i.e., with respect to pointwise convergence. (For further discussion, see [10] and [11].) 


\section{B. Sampling}

Let $\mathcal{X}$ be the (locally convex) space of functions of interest, which are defined over a domain $\Omega \subset \mathbb{R}^{d}, d \geq 1$. Given $f \in \mathcal{X}$ and a functional $\lambda \in \mathcal{X}^{*}$, we refer to the quantity $\lambda f$ as the sample of $f$ at $\lambda$. Thus, for example, when $f$ belongs to the space of rapidly decreasing smooth functions (the Schwartz space), $\delta_{5} f:=f(5)$ is the point-sample of $f$ at $5 . \int_{-\infty}^{+\infty} f(t) e^{j \omega t} \mathrm{~d} t$ is also a sample of $f$, namely, the frequency sample at frequency $\omega$. In this case, the target field $\mathbb{F}$ is that of complex numbers.

\section{Discrete Signals}

A set of values in $\mathbb{F}$ associated with members of a linearly independent set $\Lambda \subset \mathcal{X}^{*}$ can be viewed as a discrete signal, say, $a$, that lives in $\mathbb{F}^{\Lambda}$ (the set of maps from $\Lambda$ to $\mathbb{F}$ ) and can be represented by the values $a[\lambda], \lambda \in \Lambda$.

We are interested in discrete signals obtained by sampling a function $f \in \mathcal{X}$, in which case we will have $a[\lambda]:=\lambda f$. This formulation allows us to keep track of not only the sample values (i.e., $a[\lambda]$ 's) but also of the sampling procedure that provides these samples. This extra information is captured in the formulation of the set $\Lambda$ of sampling functionals. (de Boor [8], following G. Birkhoff, calls the map from $f$ to its samples over $\Lambda$ a data-map).

By $\mathcal{S}(\Lambda)$, we denote the space of those discrete signals defined on $\Lambda$ that are obtained by sampling functions in $\mathcal{X} . a \in$ $\mathcal{S}(\Lambda)$ may be restricted to a nonempty $\Xi \subset \Lambda$ to represent a signal in $\mathcal{S}(\Xi)$, for which we use the same symbol $a$.

\section{Perfect Reconstruction}

When the function space $\mathcal{X}$ in consideration is that of bandlimited signals, the Whittaker-Nyquist-Shannon sampling theorem provides conditions for perfect recovery of a function from its uniform point-evaluations. Similar conditions can be derived for other classes of functions and also for settings involving irregular sampling and sampling schemes that may not correspond to point-evaluations. A generalization is provided by the following theorem and its corollary. We do not replicate the proof of the theorem due to space limitations. It may be found in [11].

Theorem 1 (Generalized Sampling): A discrete signal $a \in$ $\mathbb{F}^{\Lambda}$, with $\Lambda \subset \mathcal{X}^{*}$ linearly independent, identifies at most one function $f$ in $\mathcal{X}$ satisfying

$$
\lambda f=a[\lambda], \quad \text { for all } \lambda \in \Lambda
$$

if and only if span $\Lambda$ is weakly-* dense in $\mathcal{X}^{*}$.

Corollary 1: With the same conditions as in theorem 1, $a \in$ $\mathcal{S}(\Lambda) \subset \mathbb{F}^{\Lambda}$ identifies exactly one $f \in \mathcal{X}$ satisfying (1).

Proof: Since $\mathcal{S}(\Lambda)$ consists of signals obtained by sampling functions in $\mathcal{X}$, there should already be at least one $f$ satisfying (1). Its uniqueness then follows from theorem 1.

\section{RESAMPING FILTERS}

Introduction of the promised resampling filters further requires a formal formulation of the functional interpolation problem.

\section{A. Functional Interpolation}

Given a space $\mathcal{X}$ and a signal $a \in \mathcal{S}(\Lambda)$ with $\Lambda \subset \mathcal{X}^{*}$, a functional interpolation problem is concerned with finding a func- tion $q_{a}$ in a subspace $\mathcal{Q}$ (the space of interpolating functions) of $\mathcal{X}$, such that

$$
\lambda q_{a}=a[\lambda], \quad \text { for all } \lambda \in \Lambda \text {. }
$$

$q_{a}$ is then known as an interpolant. Notice that this is a generalization of Lagrange interpolation, which addresses the interpolation of point-evaluations using polynomials.

For a finite $\Lambda$, it is often of interest to characterize a (finite-dimensional) subspace $\mathcal{Q}_{\Lambda}$ of $\mathcal{Q}$, such that for any arbitrary signal $a \in \mathcal{S}(\Lambda)$, there is exactly one interpolant in $\mathcal{Q}_{\Lambda}$, in which case it is said that $\mathcal{Q}_{\Lambda}$ is correct for $\Lambda$.

Let $B_{\mathcal{Q}_{\Lambda}}:=\left\{\hat{q}_{i} \mid i \in I \subset \mathbb{Z}\right\}$ be a basis for $\mathcal{Q}_{\Lambda}$ (with $I$ finite). A unique $q_{a}$ that solves (2) exists in $\mathcal{Q}_{\Lambda}$ - which we could write as $q_{a}:=\sum_{i \in I} c_{i} \hat{q}_{i}$-whenever the linear system defined by

$$
\sum_{i \in I} c_{i}\left(\lambda \hat{q}_{i}\right)=a[\lambda], \quad \text { for all } \lambda \in \Lambda
$$

has a unique solution, i.e., is invertible. This linear system (assuming some order on $\Lambda$ ) can be rewritten in matrix form

$$
\left(\lambda \hat{q}_{i}\right)_{\substack{\lambda \in \Lambda \\ i \in I}}\left(c_{i}\right)_{i \in I}=(a[\lambda])_{\lambda \in \Lambda} .
$$

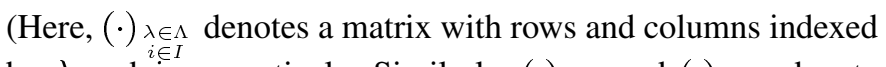
by $\lambda$ and $i$, respectively. Similarly, $(\cdot)_{i \in I}$ and $(\cdot)_{\lambda \in \Lambda}$ denote column vectors with elements respectively indexed by $i$ and $\lambda$.)

In these terms, in order to find a subspace $\mathcal{Q}_{\Lambda}$ that is correct for $\Lambda$, we can try to find a (linearly independent) subset $\left\{\hat{q}_{i} \mid i \in I\right\}$ of $\mathcal{Q}$, such that the matrix $\left(\lambda \hat{q}_{i}\right)_{\lambda \in \Lambda}$ is invertible. (One immediate consequence is that $\# \Lambda=\# I$.) We can then write

$$
\left(c_{i}\right)_{i \in I}=\left(\lambda \hat{q}_{i}\right)_{\substack{\lambda \in \Lambda \\ i \in I}}^{-1}(a[\lambda])_{\lambda \in \Lambda},
$$

and the interpolant will be

$$
q_{a}=\sum_{i \in I} c_{i} \hat{q}_{i}=\left(\hat{q}_{i}\right)_{i \in I}^{T}\left(c_{i}\right)_{i \in I}
$$

This allows us to define $\mathcal{Q}_{\Lambda}:=\operatorname{span}\left\{\hat{q}_{i} \mid i \in I\right\}$.

\section{B. Differences Between Unidimensional and Multidimensional Cases}

It is a preliminary result that for values given on any set of $n$ distinct points in $\mathbb{R}$, we can always find a unique polynomial of degree $<n$ that interpolates those values. In other words, the space $\Pi_{<n}$ of polynomials of degree less than $n$ is correct for any set of $n$ position-value pairs with distinct positions. However, in the multivariate case, it is in general no longer possible to find a space of continuous functions that is correct for all sets of $n$ conditions (for a proof, see [7]). Consequently, the polynomial interpolation problem is considerably more involved in the multivariate case. Yet, de Boor and Ron have provided an elegant solution to the functional interpolation problem (see Section III-A) using multivariate polynomials, which displays many useful properties [5], [6].

\section{C. de Boor and Ron's Least Solution}

de Boor and Ron's least solution (cf. [5]-[8]) provides a particular correct subspace of $\Pi^{d}$ for a given $\Lambda$, where $\Pi^{d}$ is the space of $d$-variate polynomials. 
Originally, de Boor and Ron formulated the least solution using a duality pairing between $\Pi^{d}$ and the space of $d$-variate formal power series [5]. For a given $\Lambda$, they defined a special subspace $\Lambda_{\downarrow}$ of $\Pi^{d}$ and proved that it is correct for $\Lambda$ and that the interpolant provided by this solution is of lesser or equal degree, if compared to any other polynomial solution.

In subsequent papers (see [6] and [8]), they showed that applying Gaussian elimination by segments to the generalized Vandermonde matrix

$$
\left(\lambda X^{\alpha}\right) \underset{\substack{\lambda \in \Lambda \\ \alpha \in \mathbb{Z}_{+}^{d}}}{\substack{d \\(n)}}
$$

(where $\mathbb{Z}_{+}^{d}$ is the set of nonnegative integer $d$-tuples, and $X^{\alpha}$ is the $d$-variate monomial of degree $\alpha$, i.e., $\left.\prod_{0 \leq i<d} x_{i}^{\alpha_{i}}\right)$ makes it possible to systematically find the space of solutions. Gaussian elimination by segments is an extension of Gaussian elimination that proceeds in a segment-by-segment (as opposed to the usual column-by-column) fashion, where a segment is a collection of adjacent columns (here formed by grouping together columns corresponding to monomials of the same degree). The result of Gaussian elimination by segments is a matrix in block rowechelon form, from which an invertible matrix $\left(\lambda \hat{q}_{i}\right)_{\substack{\lambda \in \Lambda \\ i \in I}}$ (and the basis $\left\{\hat{q}_{i} \mid i \in I\right\}$ ) can be formed (cf. [8]).

Several interesting properties of the least solution (such as monotonicity, degree-reduction, and translation invariance) are detailed in [5].

\section{D. de Boor-Ron Resampling Filters}

We are now finally in the position to introduce de Boor-Ron resampling filters. Given a space $\mathcal{S}(\Lambda)$ of signals defined over a set $\Lambda$ of sampling functionals $\lambda$, the de Boor-Ron filter $\mathfrak{P}_{\Lambda}$ is a map

$$
\mathfrak{P}_{\Lambda}: \mathcal{S}(\Lambda) \longrightarrow \mathcal{X}^{* *}
$$

(where $\mathcal{X}^{* *}$ is the second dual of the space $\mathcal{X}$ of signals; i.e., any element of $\mathcal{X}^{* *}$ assigns a numerical value to any given sampling functional $\gamma \in \mathcal{X}^{*}$ ) that is defined, for an arbitrary signal $a \in$ $\mathcal{S}(\Lambda)$, by

$$
\left(\mathfrak{P}_{\Lambda} a\right)[\gamma]:=\gamma \pi_{a}, \quad \text { for } \gamma \in \mathcal{X}^{*}
$$

where $\pi_{a}$ is the de Boor-Ron interpolant to $a[\lambda], \lambda \in \Lambda$, satisfying (2).

In simpler terms, $\mathfrak{P}_{\Lambda}$ takes a signal as its argument, interpolates it by a multivariate polynomial that lies in the de Boor-Ron space $\Lambda_{\downarrow}$ defined in [5] (and also in Section III-C), and next samples the interpolant at a given sampling functional $\gamma$. This allows us to resample a signal $a \in \mathcal{S}(\Lambda)$, that is defined on a set $\Lambda \subset \mathcal{X}^{*}$, at any given functional $\gamma$.

As we saw in (3), the interpolant depends linearly on the signal $a$. Therefore, the de Boor-Ron filter $\mathfrak{P}_{\Lambda}$ is also a linear map that can be implemented using weighted averaging (hence the name de Boor-Ron filter).

Specifically, let $\left\{\hat{q}_{i} \mid i \in I\right\}$ be a basis for the space $\mathcal{Q}_{\Lambda}:=\Lambda_{\downarrow}$ defined by de Boor and Ron's least solution. For a given signal $a \in \mathcal{S}(\Lambda)$, the interpolant $\pi_{a}$ then satisfies (3), (4)

$$
\pi_{a}=\left(\hat{q}_{i}\right)_{i \in I}^{T}\left(c_{i}\right)_{i \in I}=\left(\hat{q}_{i}\right)_{i \in I}^{T}\left(\lambda \hat{q}_{i}\right)_{\substack{\lambda \in \Lambda \\ i \in I}}^{-1}(a[\lambda])_{\lambda \in \Lambda}
$$

and therefore, for $\gamma \in \mathcal{X}^{*}$, we have

$$
\gamma \pi_{a}=\left(\gamma \hat{q}_{i}\right)_{i \in I}^{T}\left(c_{i}\right)_{i \in I}=\left(\gamma \hat{q}_{i}\right)_{i \in I}^{T}\left(\lambda \hat{q}_{i}\right)_{\substack{\lambda \in \Lambda \\ i \in I}}^{-1}(a[\lambda])_{\lambda \in \Lambda} .
$$

Let

$$
w_{\gamma}^{T}:=\left(\gamma \hat{q}_{i}\right)_{i \in I}^{T}\left(\lambda \hat{q}_{i}\right)_{\substack{\lambda \in \Lambda \\ i \in I}}^{-1}
$$

The filter $\mathfrak{P}_{\Lambda}$ is then fully specified for each $\gamma$ by the vector $w_{\gamma}$ of weights

$$
\left(\mathfrak{P}_{\Lambda} a\right)[\gamma]=w_{\gamma}^{T} \cdot(a[\lambda])_{\lambda \in \Lambda} .
$$

It may be worth mentioning that the rightmost term in (6) (i.e., $\left.\left(\lambda \hat{q}_{i}\right)_{\substack{x \in \Lambda \\ i \in I}}^{-1}\right)$ can be considered a change of basis and is equal to the identity matrix when the basis $\left\{\hat{q}_{i}\right\}$ is biorthogonal to $\Lambda$, that is, when for each $\lambda \in \Lambda, \lambda \hat{q}_{i}$ is zero except for a single $i$, for which it is equal to 1 . In the case of Lagrange interpolation, this condition translates to $\left\{\hat{q}_{i}\right\}$ being an interpolating basis.

Also, it can be immediately seen that Neville filters, introduced in [3], are special cases of de Boor-Ron filters, for settings where $\Lambda$ consists only of point-evaluation functionals, and each $\gamma$ also corresponds to a point-evaluation.

\section{APPLICATIONS}

Classic texts on signal processing and sampling usually study point-evaluations of a function over a regular grid and, in reconstruction, most often consider the case of band-limited signals and Shannon interpolation. Still, in practice, we are frequently confronted with other situations. Our signals may be obtained, e.g., not by evaluating a function at exact points on a regular grid but instead by evaluating averages using shifted versions of an integration kernel. The grid in consideration may also be irregular. Finally, a band-limited signal model may not be the most suitable in practices such as image processing, where the existence of edges suggests a treatment using piece-wise smooth functions instead. Following are a few examples demonstrating how these points can be attended to.

\section{A. Image Resampling and Prefiltering}

A single-channel digital image can be modeled as a collection of samples $I[m, n], 0 \leq m<M, 0 \leq n<N$, of a 2-D intensity function $f(x, y)$, obtained using shifted versions of a kernel $g(x, y)$

$$
I[m, n]=\int f(x, y) g\left(x-m h_{x}, y-n h_{y}\right) \mathrm{d} x \mathrm{~d} y .
$$

Here $h_{x}, h_{y}$ are, respectively, $x$ and $y$ step-sizes.

A problem in image processing and analysis is to approximate the function values over the same or a different grid. This can be accomplished using de Boor-Ron filters.

Let us first define the linear functionals $\lambda_{m, n}$

$$
\lambda_{m, n} f:=\int f(x, y) g\left(x-m h_{x}, y-n h_{y}\right) \mathrm{d} x \mathrm{~d} y .
$$

Now, to approximate $f(u, v)$, for each point $(u, v)$, we first determine the samples to be used in the approximation. This is 
done by forming a subset $\Lambda_{u, v}$ of $\lambda_{m, n}$ 's for each $(u, v) .{ }^{1}$ Also, the signal $a$ is defined by $a\left[\lambda_{m, n}\right]:=I[m, n]$.

Let $\delta_{u, v} f:=f(u, v)$ define the functional corresponding to point-evaluation at $(u, v) .\left(\mathfrak{P}_{\Lambda_{u, v}} a\right)\left[\delta_{u, v}\right]$ then approximates $f(u, v)$. This approximation will be exact when $f \in \Lambda_{u, v \downarrow}$.

This approach can be modified by replacing $\delta_{u, v}$ with

$$
\gamma_{u, v} f:=\int f(x, y) \phi(x-u, y-v) \mathrm{d} x \mathrm{~d} y
$$

(where $\phi$ is the scaling function for a given wavelet family) to construct initialization filters for Mallat's fast wavelet transform algorithm [12].

\section{B. Super-Resolution Image Reconstruction}

In super-resolution image reconstruction [2], we are typically given several discrete images $I_{k}[m, n], 0 \leq k<K$ that are sampled from slightly modified (e.g., translated, rotated, or scaled) versions of an original intensity map $f$. In such a case, we have

$$
I_{k}[m, n]:=\int\left[f \circ T_{k}(x, y)\right] g\left(x-m h_{x}, y-n h_{y}\right) \mathrm{d} x \mathrm{~d} y
$$

where $T_{k}$ 's, $0 \leq k<K$ are one-to-one transformation maps. We start by defining

$$
\lambda_{k, m, n} f:=\int\left[f \circ T_{k}(x, y)\right] g\left(x-m h_{x}, y-n h_{y}\right) \mathrm{d} x \mathrm{~d} y
$$

for $0 \leq k<K, 0 \leq m<M$, and $0 \leq n<N$ and again use $\delta_{u, v} f:=f(u, v)$, where $(u, v)$ is drawn from a new higher resolution grid. For each point $(u, v)$, we also form a set $\Lambda_{u, v}$ consisting of $\lambda_{k, m, n}$ 's to be used in our interpolation. Then, the de Boor-Ron filter $\mathfrak{P}_{\Lambda_{u, v}}$ will be our super-resolution filter at $(u, v)$, which can be applied to the signal $a\left[\lambda_{k, m, n}\right]:=I_{k}[m, n]$, to get the estimate $\left(\mathfrak{P}_{\Lambda_{u, v}} a\right)\left[\delta_{u, v}\right]$ for $f(u, v)$.

\section{Data Fusion}

Data fusion can also be addressed in a similar manner within this framework. Suppose that we are provided with $K$ sets of observations, $\left\{s_{k}[n]:=\lambda_{k, n} f \mid 0 \leq n<N_{k}\right\}_{0<k<K}$, of a function $f$. The data may be fused to arrive at an approximate description of $f$ over an arbitrary set $\Gamma$ of sampling functionals $\gamma$, by first defining a set $\Lambda_{\gamma}$ of $\lambda_{k, n}$ 's for each $\gamma$, and then applying the de Boor-Ron filter $\mathfrak{P}_{\Lambda_{\gamma}}$ to the signal $a\left[\lambda_{k, n}\right]:=s_{k}[n]$, to get the sample $\left(\mathfrak{P}_{\Lambda_{\gamma}} a\right)[\gamma]$ at $\gamma$.

A similar approach can also be used to interpolate and resample scattered measurements. This may be useful, e.g., in meteorological applications.

\section{IMPLEMENTATION}

Although the above examples may appear abstract, they can be readily implemented using the Gaussian elimination method mentioned in Section III-C, which finds the interpolant $\pi_{a}$ for any given set $\Lambda$ of functionals and signal $a \in \mathcal{S}(\Lambda)$. For instance, T. Grandine has a C implementation of this Gaussian

\footnotetext{
${ }^{1}$ The set $\Lambda_{u, v}$ typically consists of sampling functionals that are close to $(u, v)$ in some sense. It may be formed such that it does not cross any edges. In the simplest case, i.e., when $\Lambda_{u, v}$ only includes the single closest neighbor to $(u, v)$, this approach will result in nearest-neighbor interpolation. A larger set of samples may be used where the samples are more correlated, that is, where $f$ is smooth.
}

elimination method, which is available from the netlib software repository [13]. A MATLAB implementation may also be found on C. de Boor's website [14]. Performing the resampling is then merely a matter of finding the inner product of the vector $\left(c_{i}\right)_{i \in I}$ of coefficients associated with the interpolant, with the vector $\left(\gamma \hat{q}_{i}\right)_{i \in I}$, for each $\gamma \in \mathcal{X}^{*}$, as outlined in (5). Sample $\mathrm{C}$ code that uses T. Grandine's implementation is available and may be requested from the authors.

\section{CONCLUSION}

In this letter, a new formalism for studying sampling and discrete signals, using the notion of functionals, was suggested. This formalism provides a general and unified framework for many signal processing tasks and makes it possible to address resampling and interpolation problems using the machinery of functional interpolation, which allows us to design and implement resampling filters that incorporate our knowledge of the underlying sampling procedure. We suggested a family of such filters that use a particular solution to the multivariate interpolation problem, introduced by de Boor and Ron. Resampling filters using other families of interpolants may be constructed similarly. We also briefly described some applications of these filters in image and signal processing and provided pointers to some available implementations.

\section{ACKNOWLEDGMENT}

The authors would like to thank Dr. R. Israel of UBC for pointing out (on the sci.math newsgroup) a topological result on which the theorem of Section II-D depends and would also like to thank the anonymous reviewers for their reviews and valuable comments.

\section{REFERENCES}

[1] $\mathrm{X}$. Wu and L. Zhang, "Improvement of color video demosaicking in temporal domain," IEEE Trans. Image Process., vol. 15, no. 10, pp. 3138-3151, Oct. 2006.

[2] S. C. Park, M. K. Park, and M. G. Kang, "Super-resolution image reconstruction: a technical overview," IEEE Signal Process. Mag., vol. 20, no. 3, pp. 21-36, May 2003.

[3] J. Kovačević and W. Sweldens, "Wavelet families of increasing order in arbitrary dimensions," IEEE Trans. Image Process., vol. 9, no. 3, pp. 480-496, Mar. 2000.

[4] P. D. Tafti, S. Shirani, and X. Wu, "Multi-dimensional average-interpolating refinement on arbitrary lattices," in Proc. IEEE ICASSP, 2005.

[5] C. de Boor and A. Ron, "The least solution for the polynomial interpolation problem," Math. Z., vol. 210, pp. 347-378, 1992.

[6] —_ "Computational aspects of polynomial interpolation in several variables," Math. Comput., vol. 58, pp. 705-727, 1992.

[7] C. de Boor, "On the error in multivariate polynomial interpolation," Appl. Numer. Math., vol. 10, pp. 297-305, 1992.

[8] _ "Gauss elimination by segments and multivariate polynomial interpolation," in Approximation and Computation: A Festschrift in Honor of Walter Gautschi, R. Zahar, Ed. Cambridge, MA: Birkhäuser Verlag, 1994, pp. 87-96.

[9] S. Lang, Analysis II, ser. Addison-Wesley Series in Mathematics. Reading, MA: Addison-Wesley, 1969.

[10] R. E. Megginson, An Introduction to Banach Space Theory, ser. Graduate Texts in Mathematics. New York: Springer-Verlag, 1998, vol. 183.

[11] P. D. Tafti, “On multi-scale refinement of discrete data," M.A.Sc. thesis, McMaster Univ., Hamilton, ON, Canada, 2005.

[12] S. Mallat, A Wavelet Tour of Signal Processing, 2nd ed. New York: Academic, 1999.

[13] T. A. Grandine, MVP, a Package Designed to Create, Evaluate, and Manipulate Multivariate Polynomials. [Online]. Available: http://www.netlib.org/a/mvp.tgz.

[14] C. de Boor, List of m-Files for Doing Least Interpolation. [Online]. Available: http://www.cs.wisc.edu/ deboor/multiint/m_files.html. 